University of Nebraska - Lincoln

DigitalCommons@University of Nebraska - Lincoln

November 1996

\title{
The Effects of an Appraisal Manipulation: Affect, Intrusive Cognitions, and Performance for Two Cognitive Tasks
}

Scott $\mathrm{H}$. Hemenover

University of Nebraska-Lincoln

Richard A. Dienstbier

University of Nebraska-Lincoln, rdienstbier2@unl.edu

Follow this and additional works at: https://digitalcommons.unl.edu/psychfacpub

Part of the Psychiatry and Psychology Commons

Hemenover, Scott H. and Dienstbier, Richard A., "The Effects of an Appraisal Manipulation: Affect, Intrusive Cognitions, and Performance for Two Cognitive Tasks" (1996). Faculty Publications, Department of Psychology. 113.

https://digitalcommons.unl.edu/psychfacpub/113

This Article is brought to you for free and open access by the Psychology, Department of at DigitalCommons@University of Nebraska - Lincoln. It has been accepted for inclusion in Faculty Publications, Department of Psychology by an authorized administrator of DigitalCommons@University of Nebraska - Lincoln. 
Published in Motivation and Emotion, Vol. 20, No. 4 (1996). Copyright (C) 1996 Plenum Publishing Corporation. Used by permission.

\title{
The Effects of an Appraisal Manipulation: Affect, Intrusive Cognitions, and Performance for Two Cognitive Tasks ${ }^{1}$
}

\author{
S. H. Hemenover ${ }^{2}$ and Richard A. Dienstbier \\ University of Nebraska-Lincoln
}

We examined the relationship between trait measures of general appraisal and test anxiety, state measures of stress appraisals, affect, and intrusive cognition, andperformance measures on two cognitive tests (mental math and Raven matrices). Participants were randomly assigned to threat, challenge, or control conditions that were created by manipulating both primary and secondary appraisals. We predicted that the threat condition would lead to more negative affect, stress appraisals, intrusive cognitions, and more errors. While our manipulated conditions led to inconsistent effects, path analyses tended to confirm predictions that negative task appraisals and trait test anxiety lead to negative affect and to intrusive cognitions, and that for mental math test performance a path from intrusive cognitions to test errors was established. Theimportance of understanding dispositional and situational variables and their interactions during stress encounters are discussed, as is future research involving the general appraisal dimension.

\footnotetext{
${ }^{1}$ The authors wish to thank the many students who acted as experimenters and helped with data entry. Thanks go to James Johnson, Ann Schuller, and Brandon Vancura for all their hard work. Special thanks go to Roger Lott for his expert performance as the main investigator in the threat and challenge conditions. An enormous debt of gratitude also goes to my wife Kathy for all her patience and support, and for her help with the audio tapes used in this study.

This study was completed as partial fulfillment of the requirements for a Master's Degree for the first author.

Portions of this work will be presented at the annual meeting of the Midwestern Psychological Association, 1997.

${ }^{2}$ Address all correspondence concerning this article to Scott Hemenover, Department of Psychology, University of Nebraska_Lincoln, Lincoln, Nebraska 68588-0308, e-mail:shh@ unlgrad1.unl.edu
} 
Impaired performance for certain tasks (e.g., anagrams) in contexts of evaluation may be related to participants' affective and cognitive experiences. Compared to participants without these characteristics, researchers have shown performance impairments among highly anxious participants (Covington \& Omelich, 1987; Mathews \& MacLeod, 1986; Mathews, May, Mogg, \& Eysenck, 1990), participants with a dispositional tendency to experience intrusive thoughts and those experiencing intrusive thoughts during testing (Sarason, 1961; Sarason, Sarason, Keefe, Hayes, \& Shearin, 1986; Sarason \& Stoops, 1978), participants experiencing a real-life stressor (Parkinson \& Rachman, 1981), and participants appraising a task as threatening (Tomaka, Blascovich, Kelsey, \& Leitten, 1993).

An influential model of emotional experience (Lazarus, 1991), which has received considerable support in the literature (Frijda, Kuipers, \& ter Schure, 1989; Smith \& Ellsworth, 1985, 1987; Smith, Haynes, Lazarus, \& Pope, 1993; Smith \& Lazarus, 1993), proposes that patterns of cognitive appraisals produce differential affective experiences. Given the strong link between negative affectivity (e.g., anxiety, fear) and intrusive cognitions that is common among poor performing participants (e.g., Sarason \& Stroops, 1978), performance impairments in contexts of evaluation may be due to negative affectivity produced by cognitive appraisals, which leads to task interference from responses such as self-deprecating cognitions, and hence poor task performance.

Therefore, threat appraisals (high personal relevance, potential loss, and low coping ability) of a task in a realistic context should produce a pattern of negative affectivity, high rates of cognitive interference, and poor performance levels, while challenge appraisals (high personal relevance, potential gain, and high coping ability) should lead to positive affectivity, low rates of cognitive interference, and good performance. To test this possibility a study was designed in which appraisals were manipulated, and appraisals, affect, and intrusive cognition patterns were observed during the performance of two tasks (mental math and Raven matrices; Raven, 1958) for which errors were also recorded.

In early attempts to manipulate appraisals (e.g., Lazarus \& Alfert, 1964; Speisman, Lazarus \& Mordkoff, 1964), participants listened to audiotaped narratives prior to, or during, stressful films (e.g., a film on woodshop safety graphically depicting several accidents was commonly used). These narratives emphasized a particular theme, encouraging participants to view the film in a particular way (e.g., intellectually, or as traumatic). These studies were successful in producing differential subjective and physiological responses to a given stressor, but they did not address task performance or intrusive cognitions. 
To fully understand the effects of appraisal on affect, cognition, and performance, we manipulated both primary and secondary appraisals. In the theoretical system developed by Lazarus, primary appraisal is an assessment of an event's personal significance (e.g., its potential for significant loss or gain) and secondary appraisal is an assessment of one's ability to use available coping resources. These two types of appraisals interact rapidly (Folkman \& Lazarus, 1985; Lazarus \& Folkman, 1984). For example, a primary appraisal that a situation is likely to lead to disgrace may cause one to focus on one's coping deficits, and that secondary appraisal of coping deficits may reinforce the primary appraisal of impending disaster. Given their mutual causality, it may be impossible to manipulate either primary or secondary appraisals without influencing the other. Thus it seemed logical to approach the questions of this research by manipulating both primary and secondary appraisals.

More recent examinations of appraisals rarely involve their manipulation and typically focus on the interaction of personality dimensions, appraisals, and health outcomes for real-life stressors (e.g., Florian, Mikulincer, \& Taubman, 1995; Jerusalem, 1992). An exception to this trend was reported by Taylor and Scogin (1992), who manipulated primary appraisal by leading participants to believe that, on an impending test, good performance would result in rewards such as financial aid (challenge condition), or that poor performance would result in a loss of extra credit from their psychology course (threat condition). Even though the results indicated that threat participants found the impending test more important than challenge participants, no manipulation of secondary appraisal was conducted, and no test was actually performed.

In this research we were most interested in comparing two conceptually unique combinations of primary and secondary appraisals (i.e., threat: potential loss with poor coping resources; and challenge: potential benefit with good coping resources). Therefore in our manipulations we formed a threat, a challenge, and a control condition. In order to manipulate primary appraisal, we used a modification of the Taylor and Scogin procedure (1992), with secondary appraisal manipulated by providing success or failure feedback. For a task ostensibly measuring intelligence, threat-condition participants were warned about negative consequences for poor performance, and received failure feedback; challenge-condition participants were promised positive consequences for high performance, and received success feedback; and for control-condition participants performance consequences were not mentioned nor was any performance feedback given. Next, appraisal, affect, and intrusive cognition patterns were observed during two tasks (mental math and Raven matrices; Raven, 1958) for which errors were also recorded. 
In keeping with the tradition of examining the interactive relationships between stable personality dimensions and stress appraisals (e.g., Jerusalem, 1992), this study also examined two personality dimensions related to stress, appraisals, and task performance. The stress-relevant measures were dispositional test anxiety (Sarason et al., 1986), and general appraisal tendencies (Hemenover \& Dienstbier, 1996).

Although various literatures have suggested the practical importance of attributional dispositions leading one to make causal explanations of events along dimensions of stability, locus, and globality (e.g., Peterson, Vaillant, \& Seligman, 1988), less attention has been paid to dispositional appraisal styles (i.e., the general tendency across time and situations to evaluate the stressfulness of a given situation and one's ability to utilize existing coping resources, in similar ways). Because strong support exists for the role of personality in appraisal processes, a measure of general appraisal was produced by the first author, and was included in the present study (for a discussion of personality and appraisals see Hemenover \& Dienstbier, 1996).

In the context of positive, negative, or no performance feedback following the initial "test," and potential consequences for performance on future tasks, it was predicted that (1) threat-condition participants would appraise the tasks as more important, stressful, and relevant (primary appraisal), and as harder to cope with (secondary appraisal) than challengecondition or control-condition participants; (2) threat-condition participants would experience more negative affect (e.g., fear) than either controlcondition or challenge-condition participants, who would experience more positive affect (e.g., hopeful); (3) threat-condition participants would experience more intrusive cognitions than challenge-condition or controlcondition participants; (4) threat-condition participants would make the most errors on the two tasks; and (5) similar to Rotter's $(1966,1975)$ notion that only in ambiguous situations that do not force behavior irrespective of personality will personality influence appraisal processes, we predicted that only among participants moderate (ambiguous in the sense of not being clearly high or low) on the combination of general appraisal and text anxiety would situational factors such as those present in our experimental procedure moderate appraisals, affect, cognitions, and performance. That is, we predicted that situational impacts would prevail when personality does not dominate. Therefore, for participants extreme (i.e., above or below the median) on only one but not both of these dimensions, we predicted that situational factors would significantly influence responses, but the combination of extreme high or extreme low scores on both would overwhelm the situation and lead to responses consistent with these personality dimensions. 


\section{METHOD}

\section{Participants}

Participants were 105 female freshman students in an introductory psychology course at a large, urban university in the midwest United States. ${ }^{3}$ All participants received course credit for their participation.

\section{Independent Variables}

General Appraisal. Participants completed the General Appraisal Measure (GAM) containing 21 life events (e.g., fight with roommate, death of a relative). The life events comprising the GAM were gathered by modifying several events from frequently used life event checklists (e.g., Holmes \& Rahe, 1967) as well as including several events particular to college life. In an attempt to measure primary and secondary appraisals, the participants responded to two items per event: (a) "How stressful would this event be?" (stress items), and (b) "How able would you be to cope with this event?" (cope items). Participants responded on a 9-point Likert-type scales ranging from very to not at all.

In keeping with our discussion above about the rapid interactions of primary and secondary appraisals and corresponding with previous research (Tomaka et al., 1993), the main index of the GAM, called the Appraisal Quotient (AQ), was formed by taking a ratio of the stress to cope items for each event, summed across all 21 events and averaged. The resulting AQ ratio increases in magnitude as (1) stress scores increase and (2) perceived coping scores decrease. Therefore, AQ scores are distributed along a continuum ranging from a challenge appraisal style (low perceived stress and high perceived coping ability) to a threat appraisal style (high perceived stress and low perceived coping ability). The GAM exhibited acceptable internal reliability, as did all other scales (see Table I for reliability coefficients for all scales).

Test Anxiety. The Reactions to Tests Scale (RTT; Sarason, 1984) was used to measure the dispositional tendency to experience anxiety during evaluative contexts. Participants noted whether they typically experienced a given symptom during evaluations (e.g., "I get a headache during an import-

\footnotetext{
The experimental procedure was almost 2 hours long and we were concerned that effort in the latter half of the procedure would dramatically decline. In our experience, female students are more willing to complete long arduous experimental tasks than are male students; they also made up a larger portion of the research participant pool from which to draw our sample. Therefore, we restricted our participants to females.
} 
Table I. Cronbach's Alphas for All Scales for Raven Matrices and Mental Math (Shown in Parentheses) ${ }^{a}$

\begin{tabular}{lcl}
\hline \multicolumn{1}{c}{ Scale } & Number of items & Alpha \\
\hline AQ & 21 & .87 \\
CIQ & 10 & $.78(.80)$ \\
RTT & 40 & .94 \\
Primary appraisal & 3 & $.61(.60)$ \\
Secondary appraisal & 4 & $.64(.60)$ \\
Negative affect & 6 & $.94(.93)$ \\
Positive affect & 7 & $.91(.91)$ \\
Overall negative affect & 6 & .97 \\
Overall positive affect & 7 & .95 \\
Affect-specific appraisal dimensions & & \\
$\quad$ Harm & 6 & .97 \\
Loss & 3 & .96 \\
Threat & 3 & .93 \\
Optimism & 6 & .89 \\
Relevance & 2 & .90 \\
Irrelevant & 3 & .86 \\
\hline${ }^{a}$ AQ = Appraisal Quotient from the General Appraisal Measure; CIQ \\
= Cognitive Interference Questionnaire; RTT $=$ Reactions to Test \\
Scale.
\end{tabular}

ant test"). The responses were made to the 40 items on 4-point Likert-type scales ranging from not typical to very typical. The RTT has four subscales: (1) Tension, (2) Worry, (3) Test-irrelevant thinking, and (4) Bodily symptoms. Because this study was not focused on task-irrelevant cognitions (e.g., "I thought about my weekend"), only the Tension, Worry, and Bodily Symptoms subscales were combined to produce an aggregate RTT score.

\section{Dependent Variables}

Appraisal, For two cognitive tasks, the participants responded to a theoretically derived (Lazarus, 1991) measure that assessed primary appraisal of the tasks with three items (assessing the importance, relevance, and stressfulness of the tasks) and secondary appraisal with four items (coping ability, controllability, and emotion- and problem-focused coping ability). Several other appraisal items were also included (e.g., assessing the threatening and challenging nature of the tasks). Responses were made on 11-point Likert-type scales ranging from not at all to to a great extent. 
Affect The participants responded on 7-point Likert-type scales ranging from extremely to not at all, to an affect adjective scale derived from earlier research (Folkman \& Lazarus, 1985). Two dimensions were assessed:

(1) negative affect, including the terms fearful, anxious, worried, angry, sad, and disappointed', and (2) positive affect, including the terms relieved, happy, exhilarated, pleased, confident, hopeful, and eager.

Intrusive Cognitions. The Cognitive Interference Questionnaire (CIQ; Sarason et al., 1986) assessed intrusive cognitions during task performance. Participants used 5-point Likert-type scales ranging from never to very often in responding to 22 items concerning their cognitive activity during the preceding task (e.g., "I thought about how poorly I was doing"). Only the first 10 items, which focus on task-related thoughts, were aggregated and used as an Intrusive Cognitions subscale.

\section{Tasks}

The Quick Word Test This is a 100-word synonym test that measures intelligence by assessing vocabulary (Borgatta \& Corsini, 1964) presented in a multiple-choice format.

Mental Math. The participants completed 10 addition and 10 multiplication problems presented through an audio tape. The problems were easy (single digit), medium (two digits) and hard (three digits), and the number of errors served as the dependent measure of performance.

Raven Matrices. The participants completed a set of 15 items from the Standard Progressive Matrices designed to assess culture-free intelligence (Raven, 1958), and the number of errors served as the dependent measure of performance. Each item consisted of an abstract visual pattern with a missing piece. The participants chose from a number of options to complete the visual pattern.

\section{Manipulation Checks}

Affect Appraisals. We assessed six affect appraisal dimensions using 24 items derived from earlier research (Lazarus, 1991): threat (three items: e.g., "I am in danger and might not be able to handle it ), loss (three items: e.g., "I feel a sense of loss"), harm (six items: e.g., "' feel helpless"), optimism (six items: e.g., "I can handle this difficult task"), relevance (three items: e.g., "There are important things to think about here"), irrelevance (three items: e.g., "I don't care at all what is happening here'). The participants completed these items with regard to their experience during the entire procedure, responding on 7-point Likert-type scales ranging from strongly agree to strongly disagree. 
Overall Appraisal. The participants responded on 5-point Likerttype scales ranging from very slight to very much, on nine items describing the entire procedure (e.g., stressful, tension producing).

Overall Affect. The participants responded to the Affect scale, assessing negative and positive affect described earlier, with regard to their overall affect during the entire procedure.

Suspicion Questionnaire. The participants completed a set of six free-response items concerning suspcions they might have had about theprocedure. ${ }^{4}$ The participants also rated their perceived performance and effort on a 5-point Likert-type scale ranging from very slight to very much.

\section{Procedure}

The participants from the introductory psychology course signed upfor a study on "personality and task performance" and arrived in the lab in groups of 3 to 10 individuals. Each group of participants was randomly assigned to one of the three experimental conditions (threat, challenge, or control), such that all participants in a particular group experienced the same condition. There were always two experimenters present, the first author who provided performance feedback and who randomly assigned the condition, and a research assistant. The research assistant was never told what condition each group of participants had been assigned until the procedure was completed.

Control Condition. Audiotaped instructions informed the participants that the purpose of the study was to examine new tests to be used for college admissions, and that the procedure involved filling out some personality questionnaires and completing these tests. The participants read and signed consent forms, were given packets containing all the test materials, and were encouraged to try their best.

The audio tape then presented The Quick Word Test as "a new measure of intelligence that has been found to predict success in college." After instructions and an example problem, the participants were allowed $15 \mathrm{~min}$ to complete the test. Next the participants completed the GAM and then listened to audiotaped instructions for the mental math task. The tape noted that the "ability to mentally manipulate images, including numbers, predicts success in college and intelligence," and that, for this task, ". . all work must be completed mentally, and only the final solution written down. ..." The participants worked through several example problems and then completed

${ }^{4}$ Only six participants were suspicious about the procedure and their data were not included in the analysis. 
the appraisal measure for the math task, followed by the 20 mental math problems, the affect scale, and the ClQ.

The audio tape next gave instructions for the Raven matrices, again noting that "the ability to mentally manipulate images predicts success in college and intelligence." The participants completed an example problem and then the appraisal measure for the Raven matrices. The participants next completed the problems, followed by the affect scale, the ClQ, the RTI; the six affect-specific appraisal scales, the overall appraisal and affect scales for the entire procedure, and the suspicion questionnaire. The participants were then thoroughly debriefed, thanked, and dismissed.

Threat and Challenge Conditions. Prior to the audiotaped instructions, and to strengthen the effectiveness of both the threat and challenge manipulations, a distinguished-looking confederate, introduced as a psychology professor, explained that he was the "main investigator" in the study. He explained the purpose of the study just as the tape had for the control condition, but added that the experimenters wanted to follow each subject's school performance over the next 2 years to determine how well the "new tests" predicted actual college performance. He then thanked the participants and left.

Next, using audiotaped instructions, a context was created in which subject attention was focused on potential negative or positive consequences of task performance. This context was meant to manipulate primary appraisal, resulting in later tasks being appraised as more threatening or as more challenging.

Following Taylor and Scogin's (1992) procedure, the audio tape explained that (in the threat condition) (a) a group of psychologists interested in students who do poorly on these tests might be interested in interviewing those who scored below the 60th percentile rank; (b) the study was also interested in how certain hormones are related to poor performance, and so anyone scoring below the 60th percentile rank would be asked to provide a blood sample; and (c) each student who scored below the 60th percentile rank would have a record of her poor performance forwarded to her academic advisor.

For participants in the challenge condition, the tape noted that (a) a group of psychologists interested in gifted students might be interested in interviewing individuals who scored above the 90th percentile rank; (b) various departments on campus were trying to find gifted students to work as tutors, a job that paid up to $\$ 10$ an hour, and those who scored above the 90th percentile rank might be offered an opportunity to apply for one of these positions; and that (c) each student who scored above the 90th percentile rank would have a record of her excellent performance forwarded to her academic advisor. 
The participants in both the threat and challenge conditions next completed realistic-looking permission forms asking for explicit (voluntary) consent to these procedures and then completed the Quick Word Test in the same manner as did control-condition participants. Upon completion, the participants were informed that their tests would be graded while they completed a short personality measure. While they completed the GAM, the experimenter left the room, ostensibly to grade the tests. Approximately 10 min later the experimenter returned and handed each subject a realistic performance report with a percentile rank of 54 (threat condition) or 92 (challenge condition), keeping each score hidden from other participants. The remainder of the procedure proceeded as in the control condition.

\section{RESULTS}

\section{Manipulation Check}

Mean responses on the affect dimension of relevance showed that, as expected, threat-condition and challenge-condition participants found that procedure to be more relevant than did control-condition participants [i.e., with higher numbers indicating less relevant, $M s(S D s)=7.90(3.11), 8.15$ (2.85), and

9.70 (2.70), respectively; $F(2,101)=3.80, p<.03]$. Follow-up individual planned comparisons indicated that control-condition ratings of relevance were significantly lower than ratings for both threat-condition participants and for challenge-condition participants [i.e., $t(101)=2.54, p<.01$; and $t(101)=2.19, p<.03$, respectively] .

Mean ratings of overall positive affect suggested a trend of less positive affect in the threat condition compared to the challenge and control conditions [i.e., with higher numbers indicating less affect, $M s(S D s)=26.12$ (8.30), 29.40 (9.54), and 29.54 (8.90), respectively; $F(2,102)=1.670, p>$ .21]. Mean ratings of overall negative affect indicated that, as expected, threat-condition participants had higher levels of negative affect than did challenge-condition or control-condition participants [i.e., with higher numbers indicating less affect, $\mathrm{Ms}(S D s)=$

40.34 (8.90), 44.61 (7.75), and 46.03 (6.60), respectively; $F(2,102)=5.01$, $p<.008]$. Follow-up individual planned comparisons indicated that threatcondition participants experi enced significantly more negative affect than

\footnotetext{
${ }^{5}$ 0nly three participants failed to agree to some of the procedures, and no subject denied permission for all of them.

${ }^{6}$ Special care was taken when debriefing threat and challenge participants. Cookies were offered to the participants and the research hypotheses fully explained. No participants reported being angry at being deceived and many were visibly relieved to discover that no consequences (positive or negative) would befall them as a result of their "performance."
} 
did challenge-condition or control-condition participants [i.e., $t(100)=2.25$, $P<.03$, and $t(101)=3.05, p<.003$, respectively].

Mean ratings also indicated that threat-condition participants viewed the procedure as more tension producing than did challenge-condition or control-condition participants [Le., $M s(S D s)=3.28$ (1.11), 2.52 (1.25), and 2.57 (1.04), respectively; $F(2,101)=4.94, P<.009$ ]. Follow-up planned comparisons indicated that threat-condition participants found the procedure significantly more tension producing than either challenge-condition or control-condition participants [Le., $t(101)=2.79, p<.01$, and $t(101)=2.62, p<$ .01 , respectively]. Overall, the appraisal manipulation was successful in producing appropriately different patterns of appraisal and affect across the groups.

\section{Analysis of Dependent Measures}

Primary and secondary task appraisals, posttask Positive and Negative Affect scales, intrusive cognitions, and the number of errors on the two cognitive tasks served as the dependent variables in the main analyses. Median splits were performed on scores for the General Appraisal Measure and the Reactions to Test Scale and they served as independent variables, along with condition, in a series of 2 (GAM: High or Low) x 2 (RIT: High or Low) x 3 (Condition: Threat, Challenge, Control) analyses of variance (ANOVAs).

The effects presented in Table II partially supported the first prediction that threat-condition participants would make more stressful primary appraisals of the tasks than challenge-condition or control-condition participants. During the Raven matrices, threat-condition and challenge-condition participants made more stressful primary appraisals than did controlcondition participants [Le., higher means indicate more stressful primary appraisals, $M s(S D s)=13.39$ (6.0), 13.30 (5.45), and 10.50 (4.80), respectively]. Planned simple comparisons indicated that threat-condition and challenge-condition participants made significantly more stressful primary appraisals than did control-condition participants [Le., $t(102)=2.26, P<$ .03 , and $t(102)=2.14, p<.04$, respectively].

Even though measures assessing the two stable personality dimensions were completed following the appraisal manipulation, there was no difference across conditions in either the GAM $[F(2,98)=.09, p>.13$; threat condition $M=1.53$; challenge condition $M=1.37$; control condition $M=1.69]$ or the RIT $[F(2,98)=.95, p>.40$; threat condition $M=60.94$; challenge condition $M=58.92$; control condition $M=65.19]$. 
Table II. Significant Effects from Factorial Analyses of Variance for the Raven Matrices and Mental Math Tasks ${ }^{a}$

\begin{tabular}{|c|c|c|c|}
\hline Variable & $F$ & $d f$ & $p$ \\
\hline \multicolumn{4}{|c|}{ Raven } \\
\hline \multicolumn{4}{|l|}{ Primary appraisal } \\
\hline Condition & 4.14 & $(2,93)$ & .02 \\
\hline RTT & 5.47 & $(1,93)$ & .02 \\
\hline RTT $\times$ Condition & 3.04 & $(2,93)$ & .05 \\
\hline GAM $\times$ RTT $\times$ Condition & 3.93 & $(2,93)$ & .02 \\
\hline \multicolumn{4}{|l|}{ Positive affect } \\
\hline \multicolumn{3}{|l|}{ Errors } & .008 \\
\hline Condition & 3.74 & $(2,102)$ & .03 \\
\hline \multicolumn{4}{|c|}{ Mental math } \\
\hline \multicolumn{4}{|l|}{ Primary appraisal } \\
\hline Condition & 5.01 & $(2,93)$ & .009 \\
\hline \multicolumn{4}{|l|}{ Secondary appraisal } \\
\hline GAM & 4.21 & $(1,93)$ & .04 \\
\hline \multicolumn{4}{|l|}{ Negative affect } \\
\hline RTT & 9.07 & $(1,92)$ & .003 \\
\hline GAM $\times$ Condition & 3.93 & $(2,92)$ & .02 \\
\hline
\end{tabular}

Mean response ratings for primary appraisal of the mental math task showed that, consistent with our first prediction, threat-condition participants made more stressful primary appraisals than did control-condition or challenge-condition participants [i.e., $M s(S D s)=21.11$ (5.54), 18.42 (5.42), and 17.53 (5.60), respectively]. Planned simple comparisons indicated that control-condition and challenge-condition participants had significantly lower ratings of primary appraisal than did threat-condition participants [i.e., $t(102)=2.76, p<.01$, and $t(102)=2.02, p<.05$, respectively].

Relevant to our second prediction, control-condition, threat-condition, and challenge-condition participants did not differ on negative affect for the math task [i.e., $M s(S D s)=40.85$ (9.50), 44.06 (6.88), and 43.68 (7.88), respectively; $F<1.0$ ] or for the Raven [i.e., $M s(S D s)=46.08(9.40), 47.56$ (5.35), and 49.00 (8.45), respectively; $F<1.0$ ]. Control-condition, threatcondition, and challenge-condition participants also did not differ on positive affect for the math task [i.e., $M s(S D s)=26.62$ (10.05), 27.17 (8.34), and 29.37 (9.23), respectively; $F<1.0$ ] or for the Raven [i.e., $M s(S D s)=30.00$ (11.02), 30.39 (9.00), and 27.95 (9.64), respectively; $F<1.0$ ].

Inconsistent with our third prediction that threat-condition participants would experience more intrusive cognitions during task performance than other participants, threat-condition, challenge-condition, and control-con- 
dition participants did not differ on mean ratings of intrusive cognitions during the Raven matrices [i.e., $M s(S D s)=15.17$ (5.11), 14.48 (5.40), and 15.14 (4.77), respectively; $F(2,91)<1.0]$ or on mean ratings of intrusive cognitions for the mental math task [i.e., $M s(S D s)=23.06$ (6.90), 22.06 (7.62), and $22.00(6.63)$, respectively; $F(2,91)<1.0]$.

An analysis of errors on the Raven matrices indicated a significant main effect for condition (see Table II). Follow-up planned simple comparisons indicated that threat-condition participants made significantly fewer errors following threat than did control-condition participants, contrary to our fourth prediction of poorer performance $[t(102)=2.73, P<.01]$, but not significantly fewer than challenge-condition participants [i.e., $M s(S D s)=$ 1.50 (1.20), 2.58 (2.10), and 2.09 (1.63), respectively]. An analysis of errors on the mental math task indicated no significant main effects for condition [i.e., $M s(S D s)=2.47$ (1.98), 3.11 (2.01), and 2.79 (2.13), respectively; $F(2$, $91)<1.0]$.

It is likely that the negative consequences (e.g., blood draw) in the threat-condition were more motivating than the no consequences control condition. This motivational difference may have led to the unexpected superior performance in the threat condition.

The three-way interaction (GAM x RIT x Condition) on primary appraisal found for the Raven matrices was consistent with our fifth prediction that being extreme (above or below the median) on both general appraisal and test anxiety would attenuate the effects of the appraisal manipulation (see Table III). Follow-up one-way ANOVAs across condition for participants either above the median or below the median, on both test anxiety and general appraisal, were not significant. Similar analyses conducted on participants not scoring on the same side of the median on both dimensions found significant main effects for condition, with threat-condition participants making significantly more stressful primary appraisals than challengecondition and control-condition participants.

A similar three-way interaction found for positive affect was also found. Contrary to our fifth prediction, follow-up one-way ANOVAs across condition for participants either above the median or below the median on both test anxiety and general appraisal showed that only for participants low on both test anxiety and general appraisal was there a significant difference, with challenge-condition participants experiencing significantly more positive affect than either control-condition or threat-condition participants (see Table III for cell means and significance tests).

Because our manipulations failed to reliably affect secondary task appraisals, intrusive cognitions, or task performance, we sought to examine the predicted relationships among these variables with a more sensitive analysis. We therefore collapsed the data across the conditions and performed three 
Table III. Cell Means (Standard Deviations) for Three-Way Interactions for the Raven Matrices $^{a}$

\begin{tabular}{|c|c|c|c|c|c|c|}
\hline \multirow[b]{3}{*}{ GAM } & \multicolumn{6}{|c|}{ Test anxiety } \\
\hline & \multicolumn{3}{|c|}{ Low condition } & \multicolumn{3}{|c|}{ High condition } \\
\hline & Con & Thr & Chall & Con & Thr & Chall \\
\hline \multicolumn{7}{|c|}{ Primary appraisal } \\
\hline Low & $\begin{array}{l}12.00 \\
(4.47)\end{array}$ & $\begin{array}{c}9.00 \\
(4.27)\end{array}$ & $\begin{array}{l}12.15 \\
(3.63)\end{array}$ & $\begin{array}{l}9.14^{\mathrm{b}, \mathrm{c}} \\
(4.22)\end{array}$ & $\begin{array}{l}17.00^{\mathrm{b}} \\
(5.83)\end{array}$ & $\begin{array}{l}16.67^{c} \\
(3.33)\end{array}$ \\
\hline High & $\begin{array}{l}10.44 \\
(3.58)\end{array}$ & $\begin{array}{l}15.67^{\mathrm{a}} \\
(6.60)\end{array}$ & $\begin{array}{r}7.75^{\mathrm{a}} \\
(4.43)\end{array}$ & $\begin{array}{l}10.57 \\
(5.98)\end{array}$ & $\begin{array}{l}14.17 \\
(5.32)\end{array}$ & $\begin{array}{l}15.00 \\
(7.01)\end{array}$ \\
\hline \multicolumn{7}{|c|}{ Positive affect } \\
\hline Low & $\begin{array}{l}35.67^{\mathrm{e}} \\
(7.61)\end{array}$ & $\begin{array}{l}33.00^{d} \\
(9.01)\end{array}$ & $\begin{array}{l}25.69^{\mathrm{d}, \mathrm{e}} \\
(8.48)\end{array}$ & $\begin{array}{c}25.14 \\
(11.63)\end{array}$ & $\begin{array}{l}26.29 \\
(7.76)\end{array}$ & $\begin{array}{c}32.83 \\
(10.40)\end{array}$ \\
\hline High & $\begin{array}{l}29.33 \\
(5.64)\end{array}$ & $\begin{array}{l}24.17 \\
(9.30)\end{array}$ & $\begin{array}{c}34.00 \\
(15.10)\end{array}$ & $\begin{array}{l}27.35 \\
(8.02)\end{array}$ & $\begin{array}{l}27.09 \\
(8.53)\end{array}$ & $\begin{array}{c}22.60 \\
(12.73)\end{array}$ \\
\hline
\end{tabular}

${ }^{a} \mathrm{Con}=$ control; $\mathrm{Thr}=$ threat; Chall $=$ challenge; $\mathrm{GAM}=$ General Appraisal Measure. Significantly different groups share letter superscripts: ${ }^{a} F(2,18)=3.60$, $p<.05, t(16)=2.52, p<.02 ;{ }^{b} \mathrm{c} F(2,19)=6.28, p<.009, t(17)=3.70, p<.01$, and $t(17)=2.91, p<.01$, respectively; ${ }^{\mathrm{d}, \mathrm{e}} F(2,29)=3.64, p<.04, t(27)=2.37$, $p<.03$, and $t(27)=2.09, p<.05$, respectively.

multiple-regression analyses for each task. Negative affect, intrusive cognitions, and performance on each task served as criterion variables.

Consistent with our expectations that negative appraisals would lead to negative affect, in the model predicting negative affect we entered Primary and Secondary Appraisal scales, as well as two other appraisal items (threatening, challenging) in a stepwise procedure in Step 1. We chose a stepwise procedure because there was no compelling theoretical reason to expect one, and not the other, appraisal dimension to act as a significant predictor. To examine personality contributions to negative affect, beyond that provided by task appraisals, the General Appraisal Measure (GAM) and the Reactions To Tests (RIT) were entered in Step 2.

In both the models predicting intrusive cognitions and performance, the same four appraisal dimensions were first entered stepwise, and in the final step the GAM and the RIT were entered stepwise. In only the performance model, between those two steps, we entered negative and positive affect and intrusive cognitions to examine the contributions of cognitions beyond those provided by appraisals.

As seen in Table IV, viewing the math task as highly threatening and as hard to cope with, and scoring high on a dispositional measure of test anxiety, was associated with more negative affect during that task and accounted for $40 \%$ of the variance in the full model $[F(6,90)=10.05, p<$ 
Table IV. Standardized Beta Coefficients from Hierarchical Multiple Regression Predicting Negative Affect, Intrusive Cognitions, and Errors for Mental Math and Raven Matrices ${ }^{a}$

Mental math

\begin{tabular}{|c|c|c|c|c|c|c|c|}
\hline Negative affect & $\begin{array}{c}\text { Step } \\
1 \\
2\end{array}$ & $\begin{array}{l}R^{2} \\
.37 \\
.40\end{array}$ & $\begin{array}{c}\text { Prim } \\
.22^{b} \\
.14\end{array}$ & $\begin{array}{r}\text { Sec } \\
-.27^{c} \\
-.24^{c}\end{array}$ & $\begin{array}{c}\text { Threat } \\
.27^{c} \\
.29^{c}\end{array}$ & $\begin{array}{c}\text { Test anx } \\
.22^{b}\end{array}$ & \\
\hline Intrusive cognitions & $\begin{array}{c}\text { Step } \\
1 \\
2 \\
3\end{array}$ & $\begin{array}{l}R^{2} \\
.33 \\
.48 \\
.58\end{array}$ & $\begin{array}{l}\mathrm{Sec} \\
-.28^{c} \\
-.14 \\
-.08\end{array}$ & $\begin{array}{c}\text { Threat } \\
.22^{b} \\
.10 \\
.15\end{array}$ & $-.17^{b}$ & $\begin{array}{c}\text { Neg aff } \\
.45^{c} \\
.36^{c}\end{array}$ & Test anx \\
\hline Errors & $\begin{array}{c}\text { Step } \\
1 \\
2 \\
3\end{array}$ & $\begin{array}{l}R^{2} \\
.06 \\
.19 \\
.21\end{array}$ & $\begin{array}{l}\mathrm{Sec} \\
-.23 \\
-.05 \\
-.05\end{array}$ & $<.07)$ & $\begin{array}{l}\text { Post aff } \\
-.25^{c} \\
-.26^{c}\end{array}$ & $\begin{array}{l}.23(p \\
.14\end{array}$ & e cogs \\
\hline
\end{tabular}

Raven matrices

$\begin{array}{lcccc}\text { Negative affect } & \text { Step } & R^{2} & \text { Sec } & \text { Threat } \\ & 1 & .22 & -.23^{c} & .42^{c} \\ \text { fntrusive cognitions } & 2 & .24 & -.24^{c} & .39^{c} \\ & \text { scep } & \mathbb{R}^{2} & \text { Nog adr } & \text { Tese anx } \\ & 1 & .09^{d} & & \\ 2 & .31 & .53^{c} & \\ 3 & .47 & .47^{c} & .36^{c}\end{array}$

Errors

$\begin{array}{ccccc}\text { Step } & R^{2} & \text { Chall } & \text { Threat } & \text { General appraisal } \\ 1 & .17 & -.37^{c} & .58^{c} & \\ 2 & .20 & -.15 & .49^{c} & \\ 3 & .25 & -.35^{c} & .47^{c} & .19(p<.07)\end{array}$

${ }^{a}$ Prim $=$ primary appraisal; $\mathrm{Sec}=$ secondary appraisal; Text anx $=$ test anxiety; Chall $=$ challenge appraisal; Neg aff = negative affect; Pos aff = positive affect; Intrusive cogs = intrusive cognitions. Irrespective of the actual scoring conventions described in the text, for conceptual clarity the beta coefficients are all signed so that a positive sign indicates a positive relationship between the variables (e.g., that higher threat is associated with higher negative affectivity).

$b_{p}<.05$.

${ }^{c} p<.01$.

${ }_{\text {No }}$ predictors were significant on this step.

.0001]. Viewing the math task as challenging, experiencing high rates of negative affect, and scoring higher on a dispositional measure of test anxiety predicted higher intrusive cognitions, accounting for $58 \%$ of the variance in the full model $[F(8,88)=15.43, P<.0001]$. Additionally, experiencing less positive affect and more intrusive cognitions was associated with more errors on the task, accounting for $21 \%$ of the variance in the full model $[F(9$, $97)=2.59, p<.01]$. 
Similar results were found for the Raven matrices. As seen in the second part of Table IV, viewing the Raven task as highly threatening and as hard to cope with was associated with more negative affect during that task and accounted for $24 \%$ of the variance in the full model $[F(6,90)=4.95, p$ $<.0002]$. Experiencing more negative affect, and scoring higher on a dispositional measure of test anxiety was associated with higher intrusive cognitions, accounting for $47 \%$ of the variance in the full model $[F(8,88)=$ $9.64, p<.0001]$. Additionally, viewing the Raven as less challenging and more threatening, and generally tending to view events as threatening, was associated with more errors on the Raven, accounting for $25 \%$ of the variance in the full model $[F(9,87)=3.14, p<.003]$.

These results are supportive of the predicted relationships among appraisal, affect, intrusive cognitions, and task performance, and clearly illustrate the contribution that personality made to the affective and cognitive experiences of our participants. In order to more formally test the predicted relationships among appraisal, affect, intrusive cognitions and task performance, we used the significant predictors (from the regression analyses) of negative affect, intrusive cognitions, and performance for each task to test a path model for performance on the Raven matrices, and a path model for the mental math task performance (see Fig. 1, Models a and b, respectively). Since our predictions focused on intrusive cognitions, we also included a path from intrusive cognitions to performance.

Both the model for the Raven and for mental math were acceptable fits to the observed data [i.e., $\chi 2 s(d f)$ and adjusted goodness-of-fit index or GFIs $=6.98$ (11), $p<.81, .94$; and $14.65(12), p<.26, .90)$. As seen in Fig. 1, for the Raven, there were significant paths from secondary appraisal and threat appraisal to negative affect; from threat appraisal, challenge appraisal, and general appraisal tendencies (moderately significant, $p<.11$ ), to performance; as well as from negative affect to intrusive cognitions. The path from intrusive cognitions to performance was not significant and was removed from the model.

In Fig. 1 Model $b$ for the math task, there were significant paths from secondary appraisal, threat appraisal, and dispositional test anxiety to negative affect; from challenge appraisal, negative affect, and dispositional test anxiety to intrusive cognitions; from secondary appraisal to positive affect; and from intrusive cognitions and positive affect to performance.

These results parallel and confirm the multiple-regression results, while fully supporting our predictions for the mental math task. For the Raven, our predictions were partially supported, the one exception being the nonsignificant path from intrusive cognitions to performance, which was removed from the model. 
a

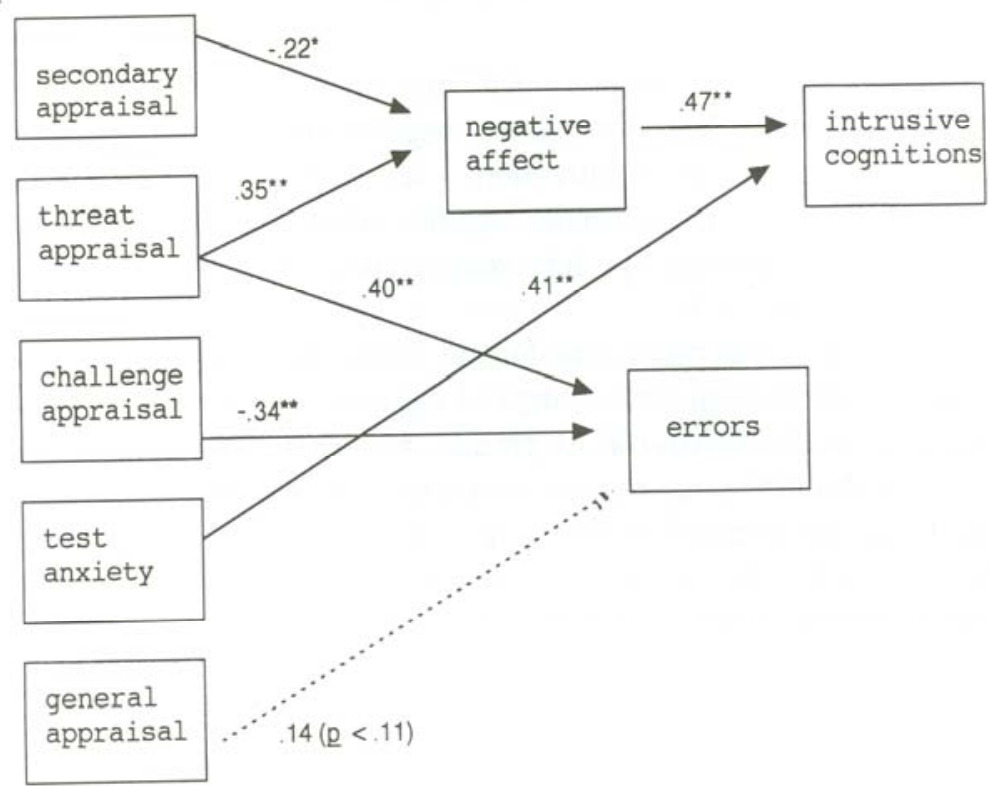

b

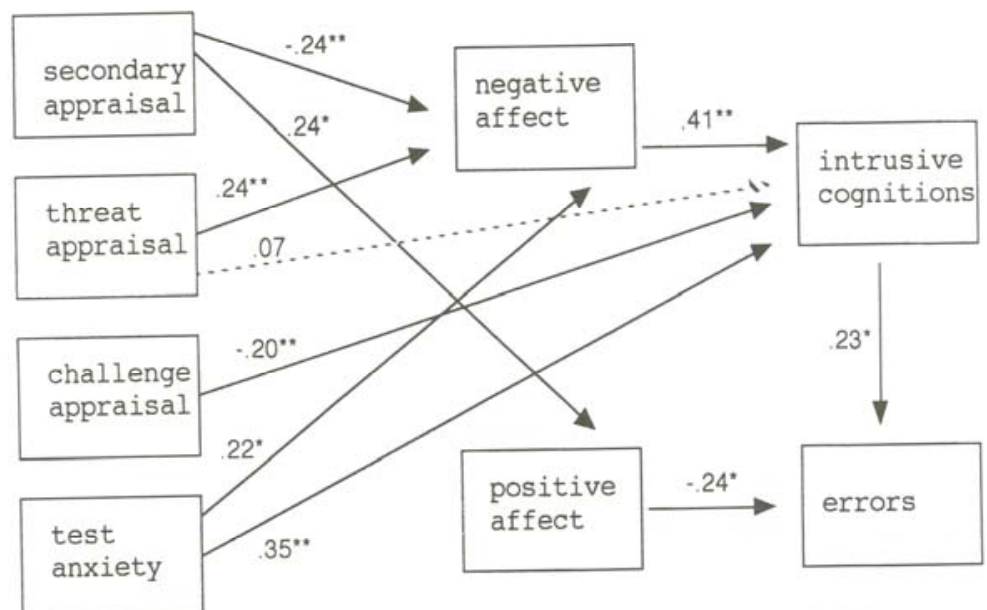

Fig. 1. Standardized path coefficients for the Raven matrices (Model a) and for the Mental Math Task (Model b). Note. Irrespective of the actual scoring conventions described in the text, for conceptual clarity the path coefficients are all signed so that a positive sign indicates a positive relationship between the variables (e.g., that higher threat is associated with higher negative affectivity). ${ }^{*} p<.05 .{ }^{* *} p<.01$. 


\section{DISCUSSION}

Moderated by several personality dimensions, and consistent with our predictions, participants warned about negative consequences for poor task performance made significantly more stressful primary appraisals for the math task, and experienced more negative affect and tension overall than did participants promised positive consequences or those not offered consequences of any kind.

Contrary to our predictions for the Raven, promise of positive consequences in the challenge condition, and negative consequences in the threat condition, produced equivalent primary appraisals. The finding that the predicted distinction on primary appraisal between threat and challenge conditions was observed for the math task, however, suggests that the failure to do so for the Raven may have been due to the timing of this task. That is, while the mental math task was completed soon after the primary appraisal manipulation, the Raven matrices were completed approximately 25 to 30 min later. Because of this, the nature of the consequences (i.e., positive or negative) may have been less salient during the Raven matrices.

The three-way interaction on primary appraisal supported our prediction that inconsistency in the personality measures of general appraisal and test anxiety would lead to between-condition appraisal differences, while being consistently high or low on both general appraisal and test anxiety would overwhelm situational influences, so there would be no betweencondition appraisal differences. This interaction resulted in equivalent scores across conditions only for participants scoring extreme on both personality dimensions.

The three-way interaction on positive affect for the Raven matrices resulted in affect differences only among those consistently low on both general appraisal and test anxiety. These results indicate that these personality dimensions may not work in an additive fashion to produce affect patterns.

While it is far more common to consider that powerful situations will overwhelm the impact of personality and fashionable to emphasize the relative weakness of personality in relationship to situations (Mischel, 1973), predicting and finding (as we did for primary appraisal) that personality dispositions may at times overwhelm situations (Le., difference caused by our manipulations of threat, challenge, and control), adds to our understanding of the relative contributions of personality and situational characteristics to stress-relevant variables (e.g., appraisal, affect).

Although we predicted that threatening participants with negative consequences would increase instances of intrusive cognitions and impair their performance on two tasks compared to other participants, we found 
no differences across conditions on intrusive cognitions. Similarly the only difference on task performance was that threat-condition participants made fewer errors on the Raven matrices than control-condition participants.

Several factors may explain these weak between-condition results. First, most participants indicated few intrusive cognitions (means for the Cognitive Intrusive Questionnaire ranged from 14.48 to 23.06, with an absolute range from 10 to 50 ). This may be because the tasks were highly believable. During the procedure most participants were observed to be fully engaged in the tasks, and during debriefing no subject reported being suspicious about the procedure. This may have sustained concentration and effort throughout the procedure, producing the observed homogeneously low rates of intrusive cognitions, which in turn may have attenuated errors.

Second, inconsistent with our predictions, manipulating performance feedback on the preliminary (Quick Word) task was not successful in inducing differential secondary appraisals of the subsequent tasks. The example problems participants completed before each of the two test tasks may have provided the participants with confidence in their ability to cope well with the tasks, so that the earlier performance feedback was discounted.

Third, the participants found the tasks easy, as seen by most threatcondition, challenge-condition, and control-condition participants correctly solving $80 \%$ to $90 \%$ of the problems. This restriction of range would prevent participants from substantially improving their performance. Thus the lack of cognitive and performance differences across groups that exhibited different primary appraisal and affect patterns may have been because of methodological problems, and not because these variables were unrelated to performance. The predicted relationships between appraisal, affect, cognition, and performance were supported by the multiple-regression analyses and by the adequate fit of the two path models.

The results form the regression analysis showed that participants who appraised the Raven matrices as highly threatening and hard to cope with experienced more negative affect while completing that task (see Table IV). Participants who experienced more negative affect during the Raven matrices, and who scored high on a measure of dispositional test anxiety, experienced more intrusive cognitions during task performance. Further results showed that participants who appraised the Raven as highly threatening and less challenging, and who disposition ally tended to view events as threatening, made more errors.

A similar pattern of results was found for the mental math ask, with participants experiencing more negative affect who appraised the task as threatening and their ability to cope with it as low, and who dispositionally tended to view tests as anxiety provoking (see Table IV). Participants ex- 
periencing more negative affect during the math task who appraised it as highly threatening and not challenging, and who disposition ally tended to view tests as anxiety provoking, experienced more intrusive cognitions during task performance. Further results showed that participants who experienced less positive affect and more intrusive cognitions during the math task made more errors.

In order to more formally test the predicted relationships among appraisal, affect, intrusive cognitions, and performance, we tested two path models (see Fig. 1). The model for the Raven matrices partially supported our predictions. As predicted, appraisals predicted affect, and affect predicted patterns of intrusive cognitions. However, intrusive cognitions did not predict performance as expected, while, unexpectedly, both task appraisals and general appraisal tendencies did.

While partially unexpected, this result is consistent with earlier findings that scoring high on dispositional test anxiety leads to poor performance (e.g., Sarason \& Stoops, 1978). Also, our finding that threat appraisals were associated with negative affect and poor performance is consistent with earlier results showing that being highly anxious (i.e., following a threat appraisal) leads to poor task performance (Mathews \& MacLeod, 1986). The findings for the math task more precisely supported our predictions. Appraisals predicted affect, and affect predicted patterns of intrusive cognitions that, along with a lack of positive affect, predicted performance.

However, the finding that intrusive cognitions predicted performance on the math task and not on the Raven may have been due to the significantly lower rate of these cognitions for the Raven compared to the math task [i.e., $M s(S D s)=14.94$ (5.05), and 22.39 (6.98), respectively; $t(102)=$ $13.40, p<.0001]$.

In the present study, methodological limitations apparently affected our ability to experimentally demonstrate appraisal-induced task performance, a finding that would be highly complementary to the multipleregression and structural equation modeling results. While these later results are supportive of our predictions, replication is needed due to the modest sample size $(N=105)$.

In future research examining appraisal and task performance, more difficult tasks should be explored to foster less restriction of range in performance. Our successful manipulation of primary appraisal replicates earlier efforts (e.g., Taylor \& Scogin, 1992), while our unsuccessful secondary appraisal manipulation indicates that factors other than performance feedback on a prior task (e.g., successful completion of example problems on the test tasks) may have determined how participants appraised their ability to cope with the two tasks. 
Even so, the finding that secondary appraisal was a significant predictor of affect in two structural equation models supports its importance in stress transactions, and replicates earlier work on appraisals and affect (e.g., Folkman \& Lazarus, 1985; Smith \& Ellsworth, 1987).

Overall these findings supported our predictions that appraisal, affect, and intrusive cognitions ought to be related to task performance. The findings also illustrated that an examination of both situational elements (e.g., whether positive or negative outcomes are likely) and stable personality dimensions (e.g., general appraisal and test anxiety) may be necessary for a complete understanding of the complex patterns of appraisals, affect, and performance during stress.

The further finding that the general appraisal dimension was useful in predicting appraisals, affect, and task performance during stress was consistent with earlier research (Hemenover \& Dienstbier, 1996) and confirms the theoretical and practical importance of that new personality dimension. These results combined with the high internal reliability of the General Appraisal Measure provide supportive evidence that it may be useful to assess this important dimension. Further research is needed to fully understand the influence of general appraisal tendencies on other aspects of the stress process (e.g., coping patterns, health outcomes).

\section{REFERENCES}

Borgatta, E. F., \& Corsini, R. J. (1964). Quick Word Test manual. New York: Harcourt, Brace, and World.

Covington, M. V., \& Omelich, C. L. (1987). Item difficulty and test performance among high anxious and low anxious students. Advances in Test Anxiety Research, 5, 127-135.

Florian, V., Mikulincer, M., \& Taubman, O. (1995). Does hardiness contribute to mental health during a stressful real life situation? The roles of appraisal and coping. Journal of Personality and Social Psychology, 68, 687-695.

Folkman, S., \& Lazarus, R. S. (1985). If it changes it must be a process: Study of emotion and coping during three stages of a college examination. Journal of Personality and Social Psychology, 48, 150-170.

Frijda, N. H., Kuipers, P., \& ter Schure, E. (1989). Relations among emotion, appraisal, and emotional action readiness. Journal of Personality and Social Psychology, 57, 212-228.

Hemenover, S. H., \& Dienstbier, R. A. (1996). Prediction of stress appraisals from mastery, extraversion, neuroticism, and general appraisal tendencies. Motivation and Emotion, 2O, 299-317.

Holmes, T. H., \& Rahe, R. H. (1967). The social readjustment rating scale. Journal of Psychosomatic Research, 11, 213-218.

Jerusalem, M. (1992). Personal resources, environmental constraints, and adaptation processes: The predictive power of a theoretical stress model. Personality and Individual Differences, 14, 15-24.

Lazarus, R. S. (1991). Emotion and adaptation. New York: Oxford University Press. 
Lazarus, R. S., \& Alfert, E. (1964). Short-circuiting of threat by experimentally altering cognitive appraisal. Journal of Abnormal and Social Psychology, 69, 195-205. Lazarus, R. S., \& Folkman, S. (1984). Stress, appraisal and coping. New York: Springer.

Mathews, A, \& MacLeod, C. (1986). Discrimination of threat cues without awareness in anxiety states. Journal of Abnormal Psychology, 95, 131-138.

Mathews, A, May, J., Mogg, K, \& Eysenck, M. (1990). Attentional bias in anxiety: Selective search or defective filtering? Journal of Abnormal Psychology, 99, 166-173.

Mischel, W. (1973). Toward a cognitive social learning reconceptualization of personality. Psychological Review, 80, 252-283.

Parkinson, L., \& Rachman, S. (1981). Part 3-Intrusive thoughts: The effects of an uncontrived stress. Advances in Behavioral Research Therapy, 3, 111-118.

Peterson, c., Vaillant, G. E., \& Seligman, M. E. P. (1988). Pessimistic explanatory style is a risk factor for physical illness: A thirty-five-year longitudinal study. Journal of Personality and Social Psychology, 55, 23-27.

Raven, J. C. (1958). StaJldard progressive matrices-Sets A, B, C, D and E. Cambridge, England: Cambridge University Press. Rotter, J. M. (1966). Generalized expectancies for internal versus external control of reinforcement. Psychological Monographs: General and Applied, 80 (Whole No. 609).

Rotter, J. M. (1975). Some problems and misconceptions related to the construct of internal versus external control of reinforcement. Journal of Consulting and Clinical Psychology, 43, 56-67.

Sarason, I. G. (1961). The effects of anxiety and threat on the solution of a difficult task. Journal of Abnormal and Social Psychology, 62, 165-168.

Sarason, I. G. (1984). Stress, anxiety, and cognitive interference: Reactions to tests. Journal of Personality and Social Psychology, 46, 929-938.

Sarason, I. G., Sarason, B. R, Keefe, D. E., Hayes, B. E., \& Shearin, E. N. (1986). Cognitive interference: Situational determinants and trait-like characteristics. Journal of Personality and Social Psychology, 51, 215-226.

Sarason, I. G., \& Stoops, R. (1978). Test anxiety and the passage of time. Journal of Consulting and Clinical Psychology, 46, 102-109.

Smith, C. A, \& Ellsworth, P. C. (1985). Patterns of cognitive appraisal in emotion. Journal of Personality and Social Psychology, 48, 813-838.

Smith, C. A, \& Ellsworth, P. C. (1987). Patterns of appraisal and emotion related to taking an exam. Journal of Personality and Social Psychology, 52, 475-488.

Smith, C. A, Haynes, K N., Lazarus, R. S., \& Pope, L. K (1993). In search of the hot cognitions: Attributions, appraisals, and their relation to emotion. Journal of Personality and Social Psychology, 65, 916-929.

Smith, C. A, \& Lazarus, R. S. (1993). Appraisal components, core relational themes, and the emotions. Cognition and Emotion, 7, 233-269.

Speisman, J. c., Lazarus, R. S., \& Mordkoff, A (1964). Experimental reductions of stress based on ego-defense theory. Journal of Abnormal and Social Psychology, 68, 367-380.

Taylor, C. J., \& Scogin, F. (1992). Dysphoria and coping in women: The effects of threat and challenge appraisals. Journal of Social and Clinical Psychology, 11, 26-42.

Tomaka, J., Blascovich, J., Kelsey, R. M., \& Leitten, C. L. (1993). Subjective, physiological and behavioral effects of threat and challenge appraisal. Journal of Personality and Social Psychology, 65, 248-260. 